\title{
Short-term outcomes after EX-PRESS implantation versus trabeculectomy alone in patients with neovascular glaucoma
}

This article was published in the following Dove Press journal:

Clinical Ophthalmology

\author{
Yoichiro Shinohara \\ Hideo Akiyama \\ Mikiya Magori \\ Shoji Kishi
}

Department of Ophthalmology, Gunma University Graduate School of Medicine, Maebashi, Gunma, Japan
Correspondence: Yoichiro Shinohara Department of Ophthalmology, Gunma University Graduate School of Medicine, 3-39-15 Showamachi, Maebashi, Gunma 37I-85I I, Japan

Tel $+8 I 272208338$

Fax +8I 27220384 I

Email mI470200I@gunma-u.ac.jp
Background: The purpose of this study was to investigate postoperative clinical outcomes in patients with neovascular glaucoma (NVG) who underwent trabeculectomy or EX-PRESS implantation and to identify predictors of successful EX-PRESS implantation.

Methods: The study designed as a retrospective observational analysis and was set in a single tertiary center. Eighty-nine patients ( 89 eyes) with NVG, were treated using trabeculectomy alone (Trab group; $n=39$ ) or EX-PRESS implantation (EX group; $n=50$ ). All patients with a history of glaucoma surgery were excluded. The main outcome measures were postoperative intraocular pressure (IOP), visual acuity (VA), number of medications, complications, additional therapy, and success rate.

Results: The mean follow-up duration was 4.6 months in the Trab group and 4.2 months in the EX group. There was no significant difference in IOP between the groups except at 1-month follow-up $(P=0.045)$. The number of patients with early postoperative hypotony, a shallow anterior chamber, hyphema, or anterior chamber irrigation was significantly lower in the EX group than in the Trab group ( $P=0.016,0.008,0.019$, and 0.014 , respectively). The other outcomes, ie, VA, number of medications, and success rate, were similar between the two groups. In the EX group, the success rate was significantly lower in 15 patients with a history of vitrectomy compared to that in 35 patients without a history of vitrectomy $(P=0.047)$.

Conclusion: Implantation of an EX-PRESS device may be more effective and safer than trabeculectomy alone in patients with NVG. However, a history of vitrectomy may affect the surgical success rate when using EX-PRESS.

Keywords: trabeculectomy, EX-PRESS, neovascular glaucoma, surgery, tube shunt

\section{Introduction}

Neovascular glaucoma (NVG) is a refractory glaucoma that occurs in several ocular ischemic diseases including diabetic retinopathy, retinal vein occlusion, and carotid artery occlusion, causing severe pain and loss of vision. ${ }^{1}$ Treatment of NVG aims to improve ocular ischemia and reduce intraocular pressure (IOP) and includes panretinal photocoagulation, injection of an antivascular endothelial growth factor agent, and antiglaucoma medications. ${ }^{2}$ It is often difficult to reduce medically uncontrollable IOP in NVG eyes, thus necessitating trabeculectomy, implantation of an Ahmed glaucoma valve, or cyclophotocoagulation. However, several surgical complications have been observed. ${ }^{3-5}$

EX-PRESS (Alcon Laboratories, Inc., Fort Worth, TX, USA) is a small, nonvalved, stainless steel drainage device used as an alternative to standard trabeculectomy in patients with glaucoma. ${ }^{6}$ The EX-PRESS P-50 model used in this study was $2.6 \mathrm{~mm}$ long with a $50 \mu \mathrm{m}$ internal diameter. Unlike standard trabeculectomy, no iridectomy 
or sclerectomy is performed in EX-PRESS implantation, which leads to shorter operating time and reduced risk of surgical complications. Previous reports have demonstrated that EX-PRESS implantation achieved better postoperative results and was safer than trabeculectomy alone. ${ }^{6-10}$ However, subjects in most of the previous studies did not have NVG. Although a few reports showed that EX-PRESS implantation reduced IOP postoperatively in eyes with $\mathrm{NVG},{ }^{11,12}$ its therapeutic effect in patients with NVG is not fully understood. To the best of our knowledge, there have been no reports comparing the effects of EX-PRESS implantation with those of trabeculectomy alone in patients with NVG.

The aim of this study was to investigate postoperative clinical outcomes in patients with NVG who underwent trabeculectomy or EX-PRESS implantation and to identify predictors of successful EX-PRESS implantation in eyes with NVG.

\section{Methods}

\section{Patients}

We retrospectively identified 89 patients with NVG who underwent trabeculectomy or EX-PRESS implantation in the Department of Ophthalmology at the Gunma University Graduate School of Medicine, between September 2007 and September 2015. Inclusion criteria were as follows: 1) presence of iris neovascularization diagnosed using slitlamp examination or gonioscopy, 2) age range from 21 to 90 years, and 3 ) increased IOP, which could not be controlled by antiglaucoma medication alone. All patients with a history of previous glaucoma surgery were excluded. The patients were divided into two groups based on whether they underwent trabeculectomy alone (Trab group; $n=39$ ) or EX-PRESS implantation (EX group; $\mathrm{n}=50$ ). This study was approved by the institutional review board of Gunma University Hospital (No 2016-058) and conformed to the guidelines under the Declaration of Helsinki. Written informed consent was obtained from all patients for the treatment and retrospective review of their medical records.

\section{Surgical technique}

In both groups, a 50\% depth square scleral flap, measuring $\sim 2.5 \times 2.5 \mathrm{~mm}$, was created after a fornix-based incision of the conjunctiva. Sponges soaked in mitomycin $\mathrm{C}$ $(0.4 \mathrm{mg} / \mathrm{mL})$ were applied under the scleral flap and conjunctiva for 3 minutes and irrigated with $200 \mathrm{~mL}$ of physiological saline. In the EX group, a P-50 EX-PRESS device was then implanted through the puncture wound using a 25-gauge needle from the limbus toward the anterior chamber. In the
Trab group, sclerostomy and peripheral iridectomy were performed. The scleral flap and conjunctiva were sutured with 10-0 nylon in both groups. When cataract surgery was also required, phacoemulsification and aspiration combined with implantation of an intraocular lens were performed after application of mitomycin $\mathrm{C}$ to the other wounds. All patients were treated with $1.5 \%$ levofloxacin and $0.1 \%$ betamethasone for 4-8 weeks after glaucoma surgery. During the early postoperative period, laser suture lysis and needling were performed, depending on postoperative IOP and bleb morphology.

\section{Clinical outcome measures}

Postoperative data were obtained on days 1 and 3, at weeks 1 and 2, and at months 1, 3, and 6. IOP was measured using a Goldmann applanation tonometer. Best-corrected visual acuity (BCVA) was recorded as decimal values and converted to logarithm of the minimum angle of resolution (logMAR) for statistical analysis.

\section{Definitions}

Success was defined as $5 \mathrm{mmHg} \leq$ IOP $\leq 21 \mathrm{mmHg}$, with or without medications. In this study, need for laser suture lysis or needling was not considered as indication of failure of the procedure. Failure was defined as two consecutive IOP measurements outside the above-mentioned range 2 weeks after glaucoma surgery, the need for further glaucoma surgery including cyclophotocoagulation, or worsening of BCVA to no light perception. Early hypotony was classified as IOP $<5 \mathrm{mmHg}$ within 2 weeks after surgery. BCVA in eyes without form vision was defined as counting fingers (0.005), hand motion (0.0025), light perception (0.00125), or no light perception $(0.0001)$, as described previously. ${ }^{13}$

\section{Statistical analysis}

The data were represented as mean \pm SD. Two-group comparisons were performed using paired or unpaired $t$-test. Categorical data were tested for differences using chi-square or Fisher's exact test. Success of treatment was investigated using Kaplan-Meier survival analysis and log-rank test. $P<0.05$ was considered to be statistically significant. Statistical analysis was performed using SPSS version 22 (IBM Corporation, Armonk, NY, USA) and GraphPad Prism version 6 (GraphPad Software, Inc., La Jolla, CA, USA).

\section{Results}

Preoperative clinical characteristics of the patients are shown in Table 1. There were no significant differences in any of the 
Table I Baseline demographic and clinical characteristics of 89 patients with neovascular glaucoma

\begin{tabular}{|c|c|c|c|}
\hline \multirow[t]{2}{*}{ Characteristics } & \multirow{2}{*}{$\frac{\text { Trabeculectomy }}{(\mathrm{N}=39), \mathrm{n}(\%)}$} & \multirow{2}{*}{$\begin{array}{l}\text { EX-PRESS } \\
(\mathrm{N}=50), \mathrm{n}(\%)\end{array}$} & \multirow[t]{2}{*}{$P$-value } \\
\hline & & & \\
\hline Sex & & & 0.276 \\
\hline Male & $27(69.2)$ & $29(58.0)$ & \\
\hline Female & $12(30.8)$ & $21(42.0)$ & \\
\hline Age (years) & & & 0.699 \\
\hline Mean \pm SD & $63.5 \pm 15.4$ & $62.2 \pm 11.6$ & \\
\hline Range & $21-90$ & $4 \mid-83$ & \\
\hline \multicolumn{4}{|l|}{ Etiology } \\
\hline$D R$ & $24(6 \mid .5)$ & $44(88.0)$ & \\
\hline OIS & 7 (I7.9) & $2(4.0)$ & \\
\hline RVO & $4(10.3)$ & $4(8.0)$ & \\
\hline RAO & $2(5.1)$ & $0(0)$ & \\
\hline Others & $2(5.1)$ & $0(0)$ & \\
\hline $\begin{array}{l}\text { Preoperative IVB } \\
(<1 \text { month })\end{array}$ & $26(66.7)$ & $29(58.0)$ & 0.404 \\
\hline
\end{tabular}

Abbreviations: DR, diabetic retinopathy; IVB, intravitreal bevacizumab; OIS, ocular ischemic syndrome; RAO, retinal artery occlusion; RVO, retinal vein occlusion.

baseline demographics or clinical characteristics between the two groups. All patients were Japanese and exhibited rubeosis iridis in preoperative slit-lamp examination or gonioscopy. The average follow-up duration was $4.6 \pm 2.0$ months in the Trab group and 4.2 \pm 2.0 months in the EX group $(P=0.79)$.

\section{IOP and visual acuity (VA) outcomes}

Preoperative and postoperative IOP values are shown in Figure 1A. IOP values in the Trab group and the EX group were $35.1 \pm 8.6 \mathrm{mmHg}$ and $33.4 \pm 10.2 \mathrm{mmHg}$ (baseline), $16.4 \pm 11.9 \mathrm{mmHg}$ and $15.7 \pm 9.5 \mathrm{mmHg}$ (day 1), $10.6 \pm 8.3 \mathrm{mmHg}$ and $10.9 \pm 6.9 \mathrm{mmHg}$ (day 3 ), $9.5 \pm 7.5 \mathrm{mmHg}$

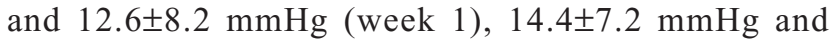
$15.8 \pm 7.6 \mathrm{mmHg}$ (week2), $18.3 \pm 9.2 \mathrm{mmHg}$ and $17.5 \pm 7.6 \mathrm{mmHg}$ (month 1), 17.9 $\pm 9.9 \mathrm{mmHg}$ and $15.8 \pm 4.7 \mathrm{mmHg}$ (month 3 ), and 15.3 $\pm 5.0 \mathrm{mmHg}$ and $17.8 \pm 6.4 \mathrm{mmHg}$ (month 6), respectively. Compared with baseline, both groups showed a statistically significant decrease in IOP at all follow-up time points $(P<0.001)$. The Trab group had a significantly lower mean IOP than the EX group at month $1(P=0.045)$. However, no statistically significant difference between the two groups was found at other time points.

As shown in Figure 1B, the mean BCVA values in the Trab group and the EX group at baseline were 1.15 logMAR and $1.24 \log$ MAR, which changed to $1.03 \log$ MAR and $1.15 \log$ MAR at 3 months, and $1.02 \log$ MAR and $1.24 \log M A R$ at 6 months, respectively. There was no statistically significant difference in VA between the two groups throughout the 6-month follow-up period. One patient in the EX group lost light perception.

\section{Required medications and success rate}

At baseline and at the final follow-up visit, the mean number of medications was $2.56 \pm 0.90$ and $0.92 \pm 1.27$, respectively, in the Trab group and $2.54 \pm 1.36$ and $1.06 \pm 1.30$, respectively, in the EX group. Both groups showed a statistically significant reduction in the number of medications at the final visit compared with that at baseline $(P<0.001$; Figure 1C). There were no significant differences in the number of medications between baseline and the final visit in either group (Figure 1C). Kaplan-Meier survival analysis of all patients showed success rates of $72.1 \%$ and $78.0 \%$ in the Trab group and the EX group, respectively, 6 months after surgery $(P=0.51$; Figure $1 \mathrm{D})$.

\section{Complications and additional interventions}

Postoperative complications observed are shown in Table 2. Early postoperative hypotony was observed in 14 (35.9\%) eyes in the Trab group and in seven (14.0\%) eyes in the EX group $(P=0.016)$. A shallow anterior chamber was observed in seven $(17.9 \%)$ eyes in the Trab group and in one $(2.0 \%)$ eye in the EX group $(P=0.019)$. Hyphema developed in $13(33.3 \%)$ eyes in the Trab group and five (10.0\%) eyes in the EX group $(P=0.008)$. Rates of other complications, including choroidal detachment, bleb leak, hypotony maculopathy, and endophthalmitis, were not significantly different between the two groups. No device-related complications were observed in the EX group.

Additional interventions are shown in Table 3. Laser suture lysis was performed as needed in $24(61.5 \%)$ eyes in the Trab group and in nine $(18.0 \%)$ eyes in the EX group $(P=0.859)$. Needling was required in $11(28.2 \%)$ eyes in the Trab group and in seven (14.0\%) eyes in the EX group $(P=0.116)$. Postoperative anterior chamber irrigation was performed more often in the Trab group (5/39 eyes) than in the EX group ( $0 / 50$ eyes; $P=0.014)$. Difference in frequency of repeat glaucoma surgery and cyclophotocoagulation between the two groups was not statistically significant $(P=0.579$ for both).

\section{Surgical risk factors for failure of EX-PRESS implantation}

No previous study has investigated surgical risk factors for failure of EX-PRESS implantation in patients with NVG. Factors potentially influencing operative success rates in this study are listed in Table 4 . We classified the 50 patients who underwent EX-PRESS implantation into the following subgroups: young age ( $<60$ years), previous vitrectomy, 


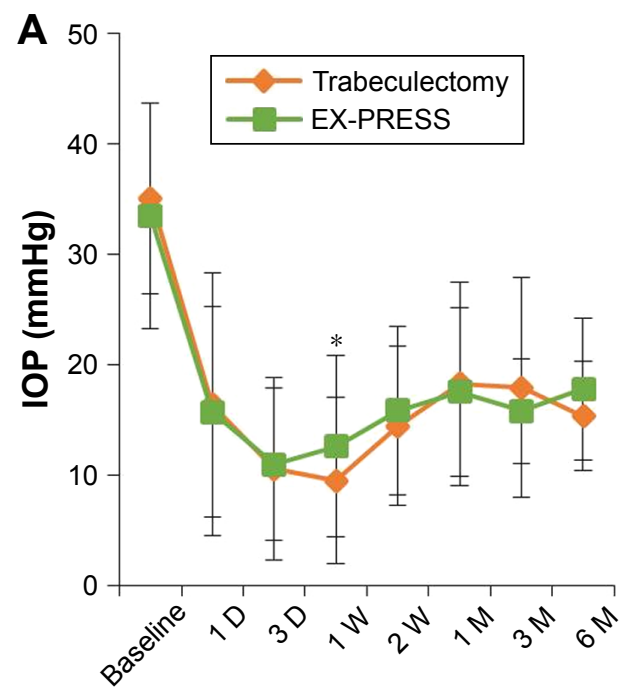

Time

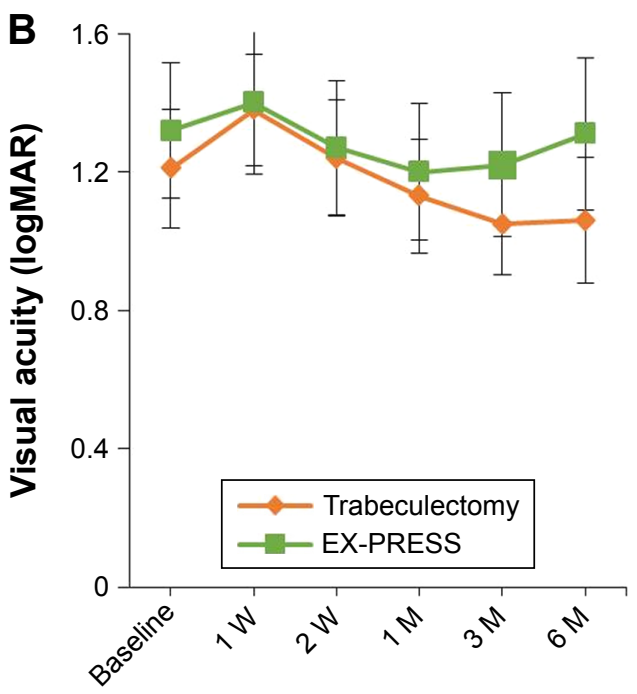

Time
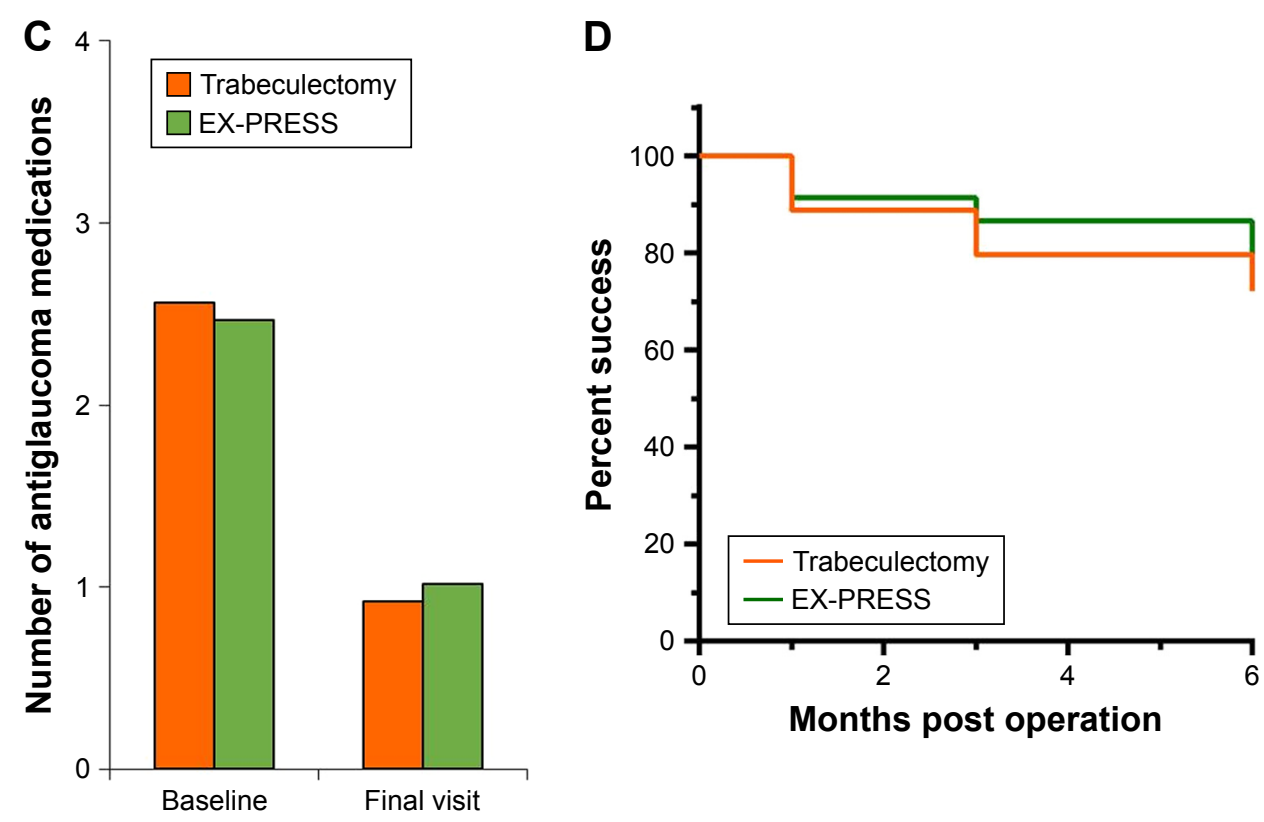

Figure I Preoperative and postoperative clinical outcomes comparing trabeculectomy alone and EX-PRESS implantation.

Notes: (A) Mean IOP was significantly reduced in both groups before and after surgery. At week I, IOP was significantly lower in the Trab group than in the EX group $(P=0.045)$. (B) Visual acuity (logMAR) after EX-PRESS implantation and trabeculectomy alone. There was no significant difference at any time point between the two groups. (C) Mean number of antiglaucoma medications was significantly lower before and after surgery in both groups. There were no significant differences between the groups at baseline and at the final visit time points. The data are expressed as mean \pm SD. (D) Kaplan-Meier survival analysis after EX-PRESS implantation versus trabeculectomy alone. Success was defined as $5 \mathrm{mmHg} \leq 1 \mathrm{IOP} \leq 2 \mathrm{I} \mathrm{mmHg}$, with or without medications. Failure was defined as follows: two consecutive IOP measurements outside the above-mentioned range 2 weeks after glaucoma surgery, repeat glaucoma surgery, or worsening of best-corrected visual acuity to no light perception. Survival rate did not differ significantly between the two groups $(P=0.5 \mathrm{I})$. $* P<0.05$.

Abbreviations: D, day; EX, EX-PRESS implantation; IOP, intraocular pressure; logMAR, logarithm of the minimum angle of resolution; M, month; Trab, trabeculectomy alone; $\mathrm{W}$, week.

preoperative intravitreal bevacizumab, and combined cataract surgery (Table 4). Kaplan-Meier survival curves for these subgroups are shown in Figure 2A-D. A history of vitrectomy was identified as a risk factor for failure of EX-PRESS implantation in patients with NVG (Table 4, Figure 2A; $P=0.047)$. We also found that young age, preoperative intravitreal bevacizumab, and combined cataract surgery were not significant predictors of the surgical outcome (Table 4; Figure 2B-D; $P=0.13,0.91$, and 0.093, respectively).

\section{Discussion}

This study compared postoperative outcomes in patients with NVG who underwent EX-PRESS implantation and those who underwent trabeculectomy. The results showed 
Table 2 Postoperative complications after trabeculectomy alone and after EX-PRESS implantation in patients with neovascular glaucoma

\begin{tabular}{|c|c|c|c|}
\hline \multirow[t]{2}{*}{ Complication } & \multirow{2}{*}{$\frac{\text { Trabeculectomy }}{(\mathrm{N}=39), \mathrm{n}(\%)}$} & \multirow{2}{*}{$\frac{\text { EX-PRESS }}{(\mathrm{N}=50), \mathrm{n}(\%)}$} & \multirow[t]{2}{*}{$P$-value } \\
\hline & & & \\
\hline $\begin{array}{l}\text { Early postoperative } \\
\text { hypotony }\end{array}$ & $14(35.9)$ & $7(14.0)$ & 0.016 \\
\hline Choroidal detachment & $9(23.1)$ & $10(20.0)$ & 0.797 \\
\hline Hyphema & $13(33.3)$ & $5(10.0)$ & 0.008 \\
\hline $\begin{array}{l}\text { Shallow anterior } \\
\text { chamber }\end{array}$ & $7(17.9)$ & I (2.0) & 0.019 \\
\hline Bleb leak (>6 weeks) & $2(5.1)$ & $2(4.0)$ & 1 \\
\hline $\begin{array}{l}\text { Hypotony } \\
\text { maculopathy }\end{array}$ & I (2.6) & $0(0)$ & 0.438 \\
\hline Endophthalmitis & $0(0)$ & $0(0)$ & N/A \\
\hline
\end{tabular}

Abbreviation: N/A, not available.

no statistically significant differences between EX-PRESS insertion and trabeculectomy alone in IOP reduction (except at 1-month follow-up), VA, number of antiglaucoma medications, or success rate. However, EX-PRESS implantation dramatically decreased rates of early postoperative hypotony, shallow anterior chamber, hyphema, and anterior chamber irrigation. In addition, the findings of this study indicated that previous vitrectomy might adversely affect the outcome of EX-PRESS implantation.

Previous studies have reported outcomes of EX-PRESS implantation but have rarely included patients with NVG. ${ }^{6-10}$ One study showed that EX-PRESS implantation combined with preoperative bevacizumab injection might be effective in patients with NVG; however, that study suffered from the limitations of a small sample size and lack of a control group. ${ }^{11}$ The EX-PRESS glaucoma filtration device versus trabeculectomy (XVT) study, ${ }^{8}$ which excluded patients with NVG, compared clinical outcomes of EX-PRESS implantation and trabeculectomy alone and found similar mean IOP, number of medications, and surgical success rates between the two groups. Moreover, the overall surgical complication

Table 3 Additional surgical treatment after trabeculectomy alone and after EX-PRESS implantation in patients with neovascular glaucoma

\begin{tabular}{|c|c|c|c|}
\hline \multirow{2}{*}{$\begin{array}{l}\text { Additional } \\
\text { intervention }\end{array}$} & \multirow{2}{*}{$\frac{\text { Trabeculectomy }}{(\mathrm{N}=39), \mathrm{n}(\%)}$} & EX-PRESS & \multirow[t]{2}{*}{$P$-value } \\
\hline & & $(\mathrm{N}=50), \mathrm{n}(\%)$ & \\
\hline Laser suture lysis & $24(6 \mid .5)$ & $9(18.0)$ & 0.859 \\
\hline Needling & II (28.2) & $7(14.0)$ & 0.116 \\
\hline $\begin{array}{l}\text { Anterior chamber } \\
\text { irrigation }\end{array}$ & $5(12.8)$ & $0(0)$ & 0.014 \\
\hline Repeat surgery & $2(5.1)$ & I (2.0) & 0.579 \\
\hline Cyclophotocoagulation & $2(5.1)$ & I (2.0) & 0.579 \\
\hline
\end{tabular}

Table 4 Potential predictors of surgical success in patients with neovascular glaucoma who underwent EX-PRESS implantation

\begin{tabular}{|c|c|c|c|}
\hline Variable & $\begin{array}{l}\text { Patients, } \\
\text { n (\%) }\end{array}$ & $\begin{array}{l}\text { Final success } \\
\text { rate, \% }\end{array}$ & $P$-value \\
\hline \multicolumn{3}{|c|}{ Age ( $<60$ years) } & \multirow[t]{3}{*}{0.13} \\
\hline Yes & $19(38.0)$ & 65.2 & \\
\hline No & $31(62.0)$ & 85.8 & \\
\hline \multicolumn{3}{|c|}{ Previous vitrectomy } & \multirow[t]{3}{*}{0.047} \\
\hline Yes & $15(30.0)$ & 55.4 & \\
\hline No & $35(70.0)$ & 81.4 & \\
\hline \multicolumn{3}{|c|}{ Preoperative IVB ( $<$ I month) } & \multirow[t]{3}{*}{0.91} \\
\hline Yes & $29(58.0)$ & 76.5 & \\
\hline No & $21(42.0)$ & 82.4 & \\
\hline \multicolumn{3}{|c|}{ Combined cataract surgery } & \multirow[t]{3}{*}{0.093} \\
\hline Yes & $12(24.0)$ & 100.0 & \\
\hline No & $38(76.0)$ & 71.4 & \\
\hline
\end{tabular}

Abbreviation: IVB, intravitreal bevacizumab.

rate was lower in the EX group than in the Trab group. Considering each surgical complication individually, rates of shallow anterior chamber and hyphema were not significantly different between the two groups in the XVT study. Additionally, a previous study showed that EX-PRESS implantation prevented early postoperative inflammation, compared with trabeculectomy. ${ }^{14}$ The main complications of trabeculectomy are attributed to overfiltration and bleeding from the iris. In the present study, no patient in the EX group required anterior chamber irrigation because of hyphema; however, five patients $(12.8 \%)$ in the Trab group required anterior chamber irrigation $(P=0.014$; Table 3$)$. Eyes with NVG are prone to develop hyphema because the iris in an eye with NVG contains many abnormal vessels, unlike an eye with primary open-angle glaucoma. Iridectomy may be disadvantageous in patients with NVG. Furthermore, early postoperative hypotony and a shallow anterior chamber were observed significantly more frequently in the Trab group than in the EX group because of overfiltration from the scleral wound. A small internal EX-PRESS area appears capable of milder and more stable filtration. The tube versus trabeculectomy (TVT) study reported that Baerveldt glaucoma implant surgery had a higher success rate than trabeculectomy in patients who had undergone previous cataract extraction with implantation of an intraocular lens and/or in patients with uncontrolled glaucoma. ${ }^{15,16}$ Although Baerveldt glaucoma implant surgery may be suitable for patients with NVG, the operating time required is longer and more advanced operator surgical skills are needed compared with trabeculectomy and EX-PRESS implantation.

An earlier study reported that previous vitrectomy was a risk factor for failure of trabeculectomy with mitomycin $\mathrm{C}$ 

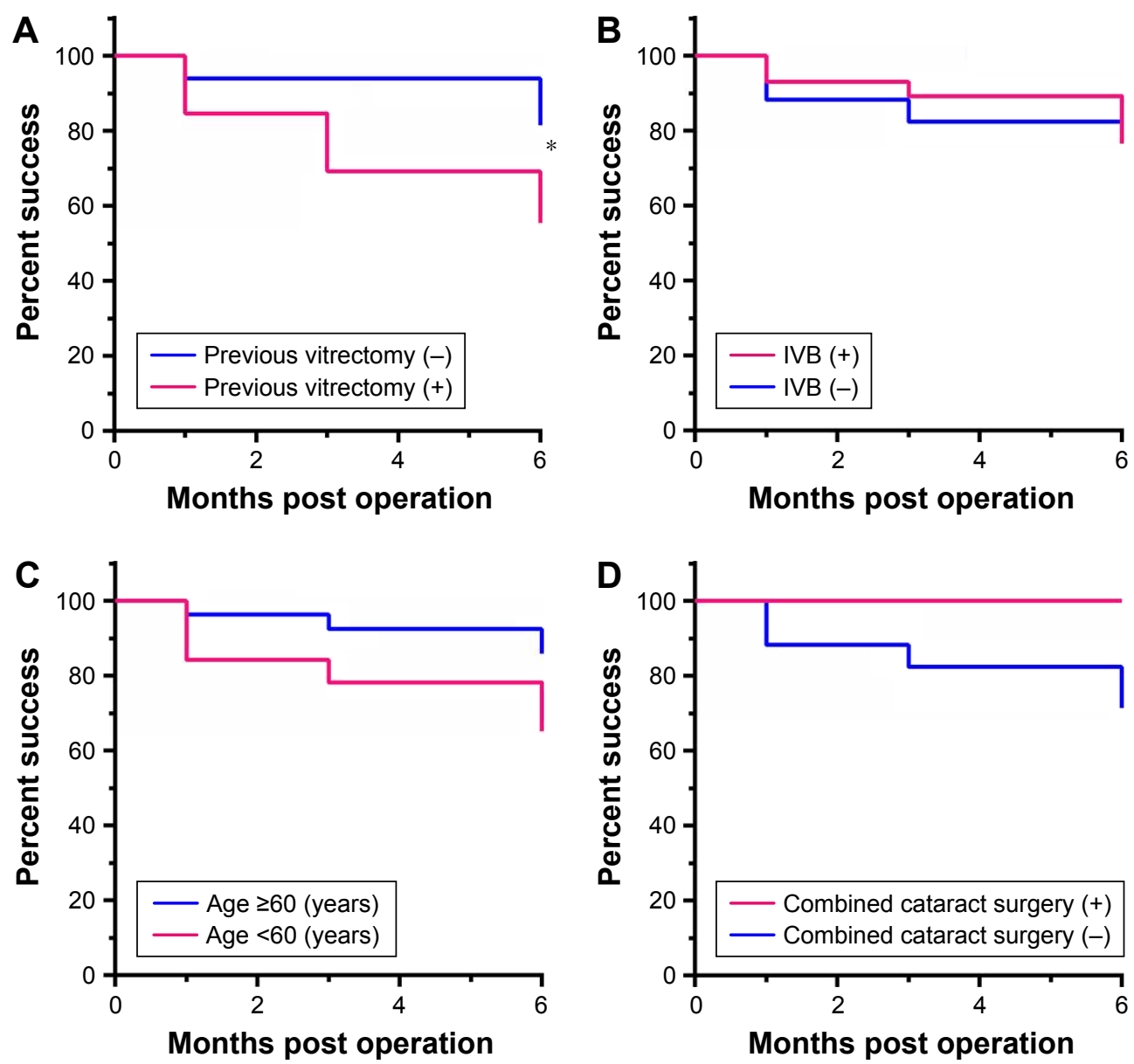

Figure 2 Kaplan-Meier survival analysis of surgical outcomes in eyes that underwent EX-PRESS implantation for neovascular glaucoma.

Notes: $(\mathbf{A})$ With a history of previous vitrectomy $(n=15)$ versus without a history of previous vitrectomy $(n=35 ; P=0.047)$. (B) With intravitreal bevacizumab $(<I$ month; $n=9)$ versus without intravitreal bevacizumab $(n=21 ; P=0.91)$. (C) Age $<60$ years $(n=19)$ versus age $\geq 60$ years $(n=31 ; P=0.13)$. (D) Combined cataract surgery $(n=12)$ versus only EX-PRESS implantation $(n=38 ; P=0.093)$. $* P<0.05$.

Abbreviation: IVB, intravitreal bevacizumab.

in patients with NVG. ${ }^{3,17}$ However, a history of vitrectomy had significant influence on operative success rates in the EX group, in this study as well. Four patients with previous vitrectomy in the EX group were defined as surgical failures because of an increase in IOP. This could be explained by the fact that vitrectomy causes diffusion of vasoformative factors, inflammation, and conjunctival scarring, as does standard trabeculectomy. ${ }^{3}$ Previous studies have reported that intravitreal bevacizumab injection may be effective in patients with NVG. ${ }^{18-20}$ We injected bevacizumab preoperatively ( $<1$ month) in 26 eyes in the Trab group and in 29 eyes in the EX group. The effect was similar, whether bevacizumab was injected preoperatively or not $(P=0.91)$. In our study, intravitreal bevacizumab was not administered if involutional neovascular vessels were clearly evident in the iris, which might explain why this treatment had no effect on the surgical success rate. Interestingly, in the present study, all patients who underwent EX-PRESS implantation in combination with cataract surgery had a successful surgical outcome ( $\mathrm{n}=12,100 \%)$. Furthermore, a previous study indicated that EX-PRESS implantation combined with phacoemulsification cataract extraction was safe and effective. ${ }^{6}$ Therefore, at the very least, combined cataract surgery might not have a negative impact on the surgical outcome when EX-PRESS implantation is performed.

The limitations of this study included its short duration of follow-up (6 months), which was because patients with excellent therapeutic effect returned to their family doctor within 12 months of surgery. Long-term operative outcomes were not assessed in this study and need to be addressed in future studies. In summary, we compared postoperative clinical outcomes in NVG after EX-PRESS implantation and those after trabeculectomy alone. Although no significant difference in surgical success rates between the two groups was found, implantation of an EX-PRESS device was significantly advantageous for prevention of postoperative overfiltration and hyphema in patients with NVG. EX-PRESS implantation may improve postoperative quality of vision 
in such patients. However, previous vitrectomy may have a negative effect on the surgical outcome after implantation of an EX-PRESS device in patients with NVG.

\section{Acknowledgments}

We are grateful to the optometrists of Gunma University for their assistance with the acquisition of the data used in this study.

\section{Disclosure}

The authors report no conflicts of interest in this work.

\section{References}

1. Brown GC, Magargal LE, Schachat A, Shah H. Neovascular glaucoma. Etiologic considerations. Ophthalmology. 1984;91(4):315-320.

2. Sivak-Callcott JA, O'Day DM, Gass JD, Tsai JC. Evidence-based recommendations for the diagnosis and treatment of neovascular glaucoma. Ophthalmology. 2001;108(10):1767-1776. quiz1777, 1800.

3. Takihara $Y$, Inatani $M$, Fukushima $M$, Iwao $K$, Iwao $M$, Tanihara $H$. Trabeculectomy with mitomycin $\mathrm{C}$ for neovascular glaucoma: prognostic factors for surgical failure. Am J Ophthalmol. 2009;147(5): 912-918, 918.e1.

4. Shen CC, Salim S, Du H, Netland PA. Trabeculectomy versus Ahmed glaucoma valve implantation in neovascular glaucoma. Clin Ophthalmol. 2011;5:281-286.

5. Netland PA, Ishida K, Boyle JW. The Ahmed glaucoma valve in patients with and without neovascular glaucoma. J Glaucoma. 2010;19(9): 581-586.

6. Traverso CE, De Feo F, Messas-Kaplan A, et al. Long term effect on IOP of a stainless steel glaucoma drainage implant (Ex-PRESS) in combined surgery with phacoemulsification. Br J Ophthalmol. 2005; 89(4):425-429.

7. Good TJ, Kahook MY. Assessment of bleb morphologic features and postoperative outcomes after Ex-PRESS drainage device implantation versus trabeculectomy. Am J Ophthalmol. 2011;151(3):507-513.e1.

8. Netland PA, Sarkisian SR Jr, Moster MR, et al. Randomized, prospective, comparative trial of EX-PRESS glaucoma filtration device versus trabeculectomy (XVT study). Am J Ophthalmol. 2014;157(2): 433-440.e3.
9. Maris PJ Jr, Ishida K, Netland PA. Comparison of trabeculectomy with Ex-PRESS miniature glaucoma device implanted under scleral flap. J Glaucoma. 2007;16(1):14-19.

10. Sugiyama T, Shibata M, Kojima S, Ueki M, Ikeda T. The first report on intermediate-term outcome of Ex-PRESS glaucoma filtration device implanted under scleral flap in Japanese patients. Clin Ophthalmol. 2011;5:1063-1066.

11. Guven Yilmaz S, Yildirim S, Degirmenci C, Ates H. Evaluation of Ex-PRESS mini glaucoma shunt implantation with preoperative intracameral bevacizumab injection in refractory neovascular glaucoma. Eur J Ophthalmol. 2016;26(6):575-580.

12. Kato N, Takahashi G, Kumegawa K, Kabata Y, Tsuneoka H. Indications and postoperative treatment for $\operatorname{Ex}-\operatorname{PRESS}((\mathrm{R}))$ insertion in Japanese patients with glaucoma: comparison with standard trabeculectomy. Clin Ophthalmol. 2015;9:1491-1498.

13. Sidoti PA, Dunphy TR, Baerveldt G, et al. Experience with the Baerveldt glaucoma implant in treating neovascular glaucoma. Ophthalmology. 1995;102(7):1107-1118.

14. Arimura S, Takihara Y, Miyake S, et al. Randomized clinical trial for early postoperative complications of Ex-PRESS implantation versus trabeculectomy: complications postoperatively of Ex-PRESS versus trabeculectomy study (CPETS). Sci Rep. 2016;6:26080.

15. Gedde SJ, Herndon LW, Brandt JD, Budenz DL, Feuer WJ, Schiffman JC. Postoperative complications in the tube versus trabeculectomy (TVT) study during five years of follow-up. Am J Ophthalmol. 2012; 153(5):804-814.e1.

16. Gedde SJ, Schiffman JC, Feuer WJ, Herndon LW, Brandt JD, Budenz DL. Treatment outcomes in the tube versus trabeculectomy (TVT) study after five years of follow-up. Am J Ophthalmol.2012;153(5): 789-803.e2.

17. Kiuchi Y, Sugimoto R, Nakae K, Saito Y, Ito S. Trabeculectomy with mitomycin $\mathrm{C}$ for treatment of neovascular glaucoma in diabetic patients. Ophthalmologica. 2006;220(6):383-388.

18. Saito Y, Higashide T, Takeda H, Ohkubo S, Sugiyama K. Beneficial effects of preoperative intravitreal bevacizumab on trabeculectomy outcomes in neovascular glaucoma. Acta Ophthalmol. 2010;88(1): $96-102$.

19. Beutel J, Peters S, Luke M, et al. Bevacizumab as adjuvant for neovascular glaucoma. Acta Ophthalmol. 2010;88(1):103-109.

20. Miki A, Oshima Y, Otori Y, Kamei M, Tano Y. Efficacy of intravitreal bevacizumab as adjunctive treatment with pars plana vitrectomy, endolaser photocoagulation, and trabeculectomy for neovascular glaucoma Br J Ophthalmol. 2008;92(10):1431-1433.
Clinical Ophthalmology

\section{Publish your work in this journal}

Clinical Ophthalmology is an international, peer-reviewed journal covering all subspecialties within ophthalmology. Key topics include: Optometry; Visual science; Pharmacology and drug therapy in eye diseases; Basic Sciences; Primary and Secondary eye care; Patient Safety and Quality of Care Improvements. This journal is indexed on Submit your manuscript here: http://www.dovepress.com/clinical-ophthalmology-journal

\section{Dovepress}

PubMed Central and CAS, and is the official journal of The Society of Clinical Ophthalmology (SCO). The manuscript management system is completely online and includes a very quick and fair peer-review system, which is all easy to use. Visit http://www.dovepress.com/ testimonials.php to read real quotes from published authors. 